\title{
Sure-wins under coherence: a geometrical perspective
}

\author{
Stefano Bonzio ${ }^{1}$ Tommaso Flaminio ${ }^{2}$ and Paolo Galeazzi ${ }^{3}$ \\ 1 Polytechnic University of the Marche \\ Via Tronto 10/a, 60126 Torrette di Ancona, Italy \\ s.bonzio@univpm.it \\ 2 Artificial Intelligence Research Institute (IIIA - CSIC) \\ Campus UAB, Bellaterra 08193, Spain \\ tommaso@iiia.csic.es \\ 3 Center for Information and Bubble Studies, University of Copenhagen \\ Karen Blixens Plads 8, 2300 København S, Denmark \\ pagale87@gmail.com
}

\begin{abstract}
In this contribution we will present a generalization of de Finetti's betting game in which a gambler is allowed to buy and sell unknown events' betting odds from more than one bookmaker. In such a framework, the sole coherence of the books the gambler can play with is not sufficient, as in the original de Finetti's frame, to bar the gambler from a sure-win opportunity. The notion of joint coherence which we will introduce in this paper characterizes those coherent books on which surewin is impossible. Our main results provide geometric characterizations of the space of all books which are jointly coherent with a fixed one. As a consequence we will also show that joint coherence is decidable.
\end{abstract}

Keywords: coherence, sure-win, de Finetti's betting game, geometry of coherence, decidability.

\section{Introduction}

The logical foundations of subjective probability theory find in the work of de Finetti, started with [1] and culminated with [2], a solid ground which, especially in the last years has been the object of a deep study and several generalizations (see for instance $[6,8,9]$ ).

To set the scene of de Finetti's apporach to probability, let us consider a bookmaker who fixes a finite number of events $e_{1}, \ldots, e_{k}$ which are represented by sentences of classical propositional logic and a book $\beta$ on them, i.e., a complete assignment $\beta:\left\{e_{1}, \ldots, e_{k}\right\} \rightarrow[0,1]$ of betting odds $\beta\left(e_{i}\right)=\beta_{i}$. In order to bet on the events, a gambler chooses stakes $\sigma_{1}, \ldots, \sigma_{k} \in \mathbb{R}$, one for each event, and pays the bookmaker the amount $\sigma_{i} \cdot \beta_{i}$ for each $e_{i}$ (with $i \in\{1, \ldots, k\}$ ). Note that $\sigma_{i}$ may be negative, in which case, paying $\sigma_{i} \cdot \beta_{i}$ means receiving $-\sigma_{i} \cdot \beta_{i}$, as money transfer is orientated from the gambler to the bookmaker. When a (classical propositional) valuation $h$ determines the truth-value of each $e_{i}$, the gambler 
gains $\sigma_{i}$ if $h\left(e_{i}\right)=1$, i.e., the event $e_{i}$ has actually occurred, and 0 otherwise ${ }^{4}$. The book $\beta$ is said to be coherent if there is no choice of stakes $\sigma_{1}, \ldots, \sigma_{k} \in \mathbb{R}$ which forces gambler's balance not to be strictly positive under every valuation $h$. In other words, coherent books are those which bar the gambler from a surewin opportunity, i.e., a strictly positive gain, independently of the truth-value of the events involved.

A slightly more general, yet completely realistic, situation is the one in which a gambler is allowed to place her stakes on two or more coherent books ${ }^{5}$. As we are going to point out in the present contribution, in such a case the sole coherence of the books the gambler decides to play with is not sufficient to bar her from a sure-win. Consider, for instance, the following very elementary example: Two bookmakers $B_{1}$ and $B_{2}$ fix betting odds to the events "Heads" and "Tails" of the typical coin-tossing game according to the following scheme: $B_{1}$ assigns $1 / 2$ to both "Heads" and "Tails", while $B_{2}$ assigns $1 / 3$ to "Heads" and $2 / 3$ to "Tails". Notwithstanding the coherence of the two assignments, buying "Tails" from $B_{1}$ for $\sigma_{1}=1$ euro and "Heads" from $B_{2}$ for $\sigma_{2}=1$ euro leads the gambler to a sure-win.

Situations of this kind which have been studied by Nau and his collaborators (see $[10,11]$ ) in the context of noncooperative games. There, given a set of players, a (conjoined) strategy is said to be jointly coherent, if it does not expose the group to arbitrage. In other words, "players who subscribe to the standard of joint coherence, are those who do not let themselves be used collectively as a money pump" (see [10, Pag. 426]).

In this paper we deepen this research line sticking within de Finetti's original betting framework and we move the first steps towards a logico-mathematical formalization of those coherent books which avoid sure-win (i.e., arbitrage) opportunities. They will be called jointly coherent books. In particular, we will give an answer to the following question: given a coherent book, which other coherent books, if any, bar a malicious gambler from a sure-win opportunity? More precisely, for every coherent book $\beta$, we will provide a geometric characterization of the set of all (coherent) books which are jointly coherent with it.

This paper is organized as follows: in the next section we will recall, in a more precise way, de Finetti's coherence criterion, de Finetti's theorem and, in particular, we will focus on its geometric version. In Section 3, we will formally introduce the concepts of sure-win and joint coherence of a book. In Section 4 we will study the geometry of joint coherence and provide the main result of the paper.

\section{Preliminaries}

Along this paper we fix a finite set of events that we denote by $\Phi=\left\{e_{1}, \ldots, e_{k}\right\}$. As we recalled in Section 1, a book $\beta$ on $\Phi$ is coherent if for each choice of stakes

\footnotetext{
${ }^{4}$ For details, see for instance [5].

5 The maybe unrealistic assumption which sees the bookmakers to consider exactly the same set of events can indeed be relaxed with an inessential modification.
} 
$\sigma_{1}, \ldots, \sigma_{k} \in \mathbb{R}$ there exists at least a possible world $h$ such that gambler's total balance $\sum_{i=1}^{k} \sigma_{i}\left(h\left(e_{i}\right)-\beta\left(e_{i}\right)\right) \leq 0$.

A book $\beta$ is said to be incoherent if it is not coherent. Obviously, incoherent books are those which allow the gambler for a sure-win opportunity.

Recall that a finitely additive probability measure over a Boolean algebra $\mathbf{A}$ is a map $P: \mathbf{A} \rightarrow[0,1]$ such that $P(1)=1$ and $P(a \vee b)=P(a)+P(b)$, provided that $a \wedge b=0$. De Finetti's theorem then states that a book $\beta: \Phi \rightarrow[0,1]$ is coherent iff it extends to a finitely additive probability measure over the Boolean algebra generated by the events in $\Phi$, denoted by $\mathbf{B}_{\Phi}$, see [2].

This result admits an equivalent geometrical formulation (see [12]), which we are going to recall here. Any finite set of events $\Phi=\left\{e_{1}, \ldots, e_{k}\right\}$ determines a polytope in $[0,1]^{k}$ by the following construction. Let $h_{1}, \ldots, h_{t}$ be the homomorphisms from $\mathbf{B}_{\Phi}$ to the two element Boolean algebra $\mathbf{2}=\langle\{0,1\}, \wedge, \vee, \neg, 0\rangle$. For every $j=1, \ldots, t$ let $q_{j}$ be the point of $\{0,1\}^{k}$

$$
q_{j}=\left(h_{j}\left(e_{1}\right), \ldots, h_{j}\left(e_{k}\right)\right) .
$$

Finally, let $\mathscr{C}_{\Phi}$ be the polytope of $[0,1]^{k}$ generated by $q_{1}, \ldots, q_{t}$ :

$$
\mathscr{C}_{\Phi}=\operatorname{co}\left(\left\{q_{j} \mid j=1, \ldots, t\right\}\right),
$$

where co stands for convex hull.

A book $\beta: \Phi \rightarrow[0,1]$ determines a point $\beta=\left(\beta\left(e_{1}\right), \ldots, \beta\left(e_{k}\right)\right) \in[0,1]^{k}$. The following result, whose proof can be found in [6, Lemma 6.3] and [12, Theorem $2]$, provides a geometric characterization of coherent books.

Theorem 1. For a book $\beta: \Phi \rightarrow[0,1]$ the following conditions are equivalent:

1. $\beta$ is coherent;

2. $\beta \in \mathscr{C}_{\Phi}$.

The construction illustrated above is better visualized towards an example.

Example 1. Consider $\Phi=\left\{e_{1}, e_{2}\right\}$, where $e_{1}=p$ and $e_{2}=p \vee q$ in a language with two propositional variables $p, q$. Following de Finetti [1], the above mentioned events may be thought as of referring to a horse race: the atomic event $p$ can be interpreted as "Horse number 1 is the winner", while $p \vee q$ could stand for "An Italian horse is winning", under the assumption that only two horses are Italian (and one of them is actually the number 1 ).

The algebra $\mathbf{B}_{\Phi}$ counts of four homomorphisms to $\mathbf{2}$, namely those maps $h_{1}, h_{2}, h_{3}, h_{4}:\{p, q\} \rightarrow\{0,1\}$ which assign, respectively, to $p$ and $q$ the values $(0,0),(0,1),(1,0)$ and $(1,1)$. Therefore, we obtain the following points $q_{1}, \ldots, q_{4} \in \mathbb{R}^{2}$ :

$$
\begin{aligned}
& q_{1}=\left(h_{1}\left(e_{1}\right), h_{1}\left(e_{2}\right)\right)=\left(h_{1}(p), h_{1}(p \vee q)\right)=(0,0) ; \\
& q_{2}=\left(h_{2}\left(e_{1}\right), h_{2}\left(e_{2}\right)\right)=\left(h_{2}(p), h_{2}(p \vee q)\right)=(0,1) ; \\
& q_{3}=\left(h_{3}\left(e_{1}\right), h_{3}\left(e_{2}\right)\right)=\left(h_{3}(p), h_{3}(p \vee q)\right)=(1,1) ; \\
& q_{4}=\left(h_{4}\left(e_{1}\right), h_{4}\left(e_{2}\right)\right)=\left(h_{4}(p), h_{4}(p \vee q)\right)=(1,1) .
\end{aligned}
$$


Since $q_{3}=q_{4}$, we have:

$$
\mathscr{C}_{\Phi}=\operatorname{co}\left(\left\{q_{1}, q_{2}, q_{3}\right\}\right)=\operatorname{co}(\{(0,0),(0,1),(1,1)\}),
$$

depicted as in Figure 1 below. Theorem 1 tells us that a book $\beta$ is coherent if and only if it is a convex combination of $q_{1}, q_{2}$ and $q_{3}$.

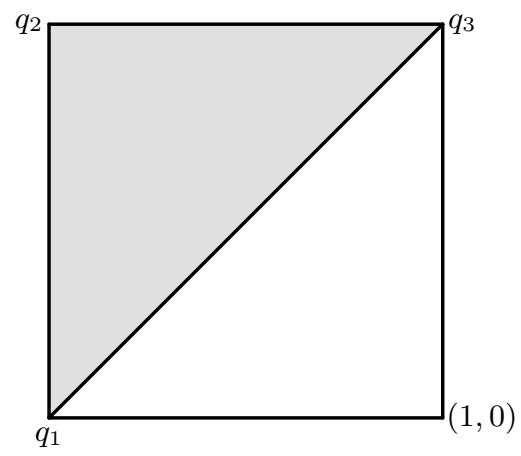

Fig. 1. The convex set $\mathscr{C}_{\Phi}$ (in gray) of all coherent books on events $e_{1}=p$ and $e_{2}=p \vee q$.

\section{$3 \quad$ Sure-wins and jointly coherent books}

As mentioned in the Introduction, we are interested in situation where a gambler has the opportunity of betting on two (or ideally more) books over the same set of events. The gambler's concept of sure-win opportunity becomes wider and it is made precise in the following.

Definition 1. Let $\beta_{1}, \beta_{2}$ be coherent books on the set of events $\Phi=\left\{e_{1}, \ldots, e_{k}\right\}$. We say that a gambler has a sure-win opportunity on $\beta_{1}, \beta_{2}$ if there exists a total map $\xi:\{1, \ldots, k\} \rightarrow\{1,2\}$ such that, the book

$$
\beta: e_{i} \mapsto \beta_{\xi(i)}\left(e_{i}\right)
$$

is incoherent. If such function $\xi$ does not exist, then $\beta_{1}$ and $\beta_{2}$ are said to be jointly coherent.

Therefore, a gambler has a sure-win opportunity on $\beta_{1}, \beta_{2}$ if there exists a map $\xi:\{1, \ldots, k\} \rightarrow\{1,2\}$ and stakes $\sigma_{1}, \ldots, \sigma_{k} \in \mathbb{R}$ such that in very every possible world $h$, gambler's balance

$$
\left.\sum_{i=1}^{k} \sigma_{i}\left(h\left(e_{i}\right)-\beta_{\xi(i)}\right)\right)>0,
$$

where $\beta: e_{i} \mapsto \beta_{\xi(i)}\left(e_{i}\right)$ is as in Definition 1. 
Remark 1. Notice that two coherent books $\beta_{1}$ and $\beta_{2}$ are jointly coherent if any book in the set

$$
\Xi\left(\beta_{1}, \beta_{2}\right)=\left\{\beta_{1}\left(e_{1}\right), \beta_{2}\left(e_{1}\right)\right\} \times\left\{\beta_{1}\left(e_{2}\right), \beta_{2}\left(e_{2}\right)\right\} \times \ldots \times\left\{\beta_{1}\left(e_{k}\right), \beta_{2}\left(e_{k}\right)\right\}
$$

is coherent as well. For any pair of coherent books $\beta_{1}, \beta_{2}$ we will call $\Xi\left(\beta_{1}, \beta_{2}\right)$ the set of crossed-books of $\beta_{1}$ and $\beta_{2}$.

Also notice that, by Definition 1, a gambler is not allowed to buy (or sell) a bet on the same event from both $\beta_{1}$ and $\beta_{2}$. This restriction is imposed in order to not trivialize her opportunities of sure-win. Indeed, since $\beta_{1}$ and $\beta_{2}$ are distinct, there always aexists at least an event $e$ such that $\beta_{1}(e) \neq \beta(e)$. Thus, assuming that $\beta_{1}(e)<\beta_{2}(e)$ without loss of generality, buying $\beta_{1}(e)$ for 1 euro and $\beta_{2}(e)$ for -1 euro (i.e., selling $\beta_{2}(e)$ for 1 euro) would immediately ensure the gambler a sure-win.

Example 2. Let $\Phi=\left\{e_{1}, e_{2}\right\}$ as in Example 1 and consider the books:

1. $\beta_{1}\left(e_{1}\right)=1 / 2$ and $\beta_{1}\left(e_{2}\right)=2 / 3$;

2. $\beta_{2}\left(e_{1}\right)=1 / 4$ and $\beta_{2}\left(e_{2}\right)=2 / 3$;

3. $\beta_{3}\left(e_{1}\right)=\beta_{3}\left(e_{2}\right)=1 / 3$.

Then, $\beta_{1}$ is jointly coherent with $\beta_{2}$, which is joint coherent with $\beta_{3}$. On the other hand $\beta_{1}$ is not jointly coherent with $\beta_{3}$. Indeed, the book $\alpha \in \Xi\left(\beta_{1}, \beta_{3}\right)$ defined by $e_{1} \mapsto \beta_{1}\left(e_{1}\right)=1 / 2, e_{2} \mapsto \beta_{3}\left(e_{2}\right)=1 / 3$ is incoherent (see Figure 2). Therefore, a gambler who is allowed to choose, for each event, which book to bet with has a sure-win opportunity if the books into play are $\beta_{1}$ and $\beta_{3}$.

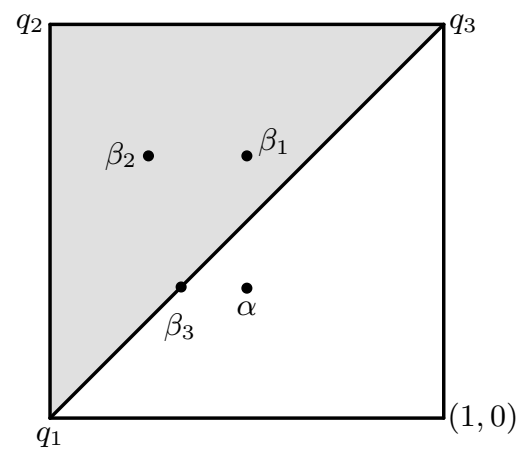

Fig. 2. The convex hull $\mathscr{C}_{\Phi}$ (gray); the coherent books $\beta_{1}, \beta_{2}, \beta_{3}$ and the incoherent book $\alpha \in \Xi\left(\beta_{1}, \beta_{3}\right)$.

We now present a first easy result. Recall that a subset $B=\left\{b_{1}, \ldots, b_{r}\right\}$ of a Boolean algebra $\mathbf{A}$ is a partition if $\bigvee_{i=1}^{r} b_{i}=\top$ and, for all $i \neq j, b_{i} \wedge b_{j}=\perp$. 
Proposition 1. If $\Phi=\left\{e_{1}, \ldots, e_{k}\right\}$ is a partition of $\mathbf{B}_{\Phi}$, then for any two coherent books $\beta_{1}, \beta_{2}$ on $\Phi$ the following conditions are equivalent:

1. $\beta_{1} \neq \beta_{2}$;

2. $\beta_{1}$ and $\beta_{2}$ are not jointly coherent, i.e. the gambler has a sure-win opportunity on $\beta_{1}, \beta_{2}$.

Proof. The direction $(2) \Rightarrow(1)$ is trivial. In order to prove $(1) \Rightarrow(2)$, observe that, since by hypothesis $\Phi$ is a partition of $\mathbf{B}_{\Phi}$, any book $\beta$ on $\Phi$ is coherent if and only if

$$
\sum_{i=1}^{k} \beta\left(e_{i}\right)=1 .
$$

Now, since $\beta_{1} \neq \beta_{2}$, there exists $e_{i} \in \Phi$ such that $\beta_{1}\left(e_{i}\right) \neq \beta_{2}\left(e_{i}\right)$. Let us assume, without loss of generality, that $\beta_{1}\left(e_{i}\right)<\beta_{2}\left(e_{i}\right)$ and let us consider the book $\beta: \Phi \rightarrow[0,1]$ defined as follows: for every $e \in \Phi$,

$$
\beta(e)= \begin{cases}\beta_{1}(e) & \text { if } e \neq e_{i}, \\ \beta_{2}(e) & \text { otherwise } .\end{cases}
$$

Notice that $\beta \in \Xi\left(\beta_{1}, \beta_{2}\right)$ and it is not coherent since $\sum_{j=1}^{k} \beta\left(e_{j}\right)<1$. Therefore, $\beta_{1}$ and $\beta_{2}$ are not jointly coherent. This settles the claim.

\section{The geometry of joint coherence}

We are interested in providing a full characterization of all those coherent books which are jointly coherent with a fixed one. In this section, we will give geometric characterizations of the space of these books.

We set the background for proving this result. For every book $\beta: \Phi \rightarrow[0,1]$ and for every $i=1, \ldots, k$, let $\delta_{i}$ be the pair $\left(d_{i}^{+}, d_{i}^{-}\right) \in \mathbb{R}^{2}$ be such that:

1. $d_{i}^{ \pm} \geq 0$;

2. the books $\beta_{d_{i}^{+}}=\left(\beta_{1}, \ldots, \beta_{i-1}, \beta_{i}+d_{i}^{+}, \beta_{i+1}, \ldots, \beta_{k}\right)$ and $\beta_{d_{i}^{-}}=\left(\beta_{1}, \ldots, \beta_{i-1}\right.$, $\left.\beta_{i}-d_{i}^{-}, \beta_{i+1}, \ldots, \beta_{k}\right)$ are coherent;

3. for all $\varepsilon>0,\left(\beta_{1}, \ldots, \beta_{i-1}, \beta_{i}+d_{i}^{+}+\varepsilon, \beta_{i+1}, \ldots, \beta_{k}\right)$ and $\left(\beta_{1}, \ldots, \beta_{i-1}, \beta_{i}-\right.$ $\left.d_{i}^{-}-\varepsilon, \beta_{i+1}, \ldots, \beta_{k}\right)$ are incoherent.

Let us hence define the rectangle

$$
\mathscr{R}_{\beta}=\left\{\gamma \in \mathbb{R}^{k}\left|(\forall i=1, \ldots, n) d_{i}^{-} \leq\right| \gamma_{i}-\beta_{i} \mid \leq d_{i}^{+}\right\},
$$

and the convex set

$$
\mathscr{C}_{\beta}=\mathscr{C}_{\Phi} \cap \mathscr{R}_{\beta} .
$$

Obviously $\mathscr{C}_{\beta}$ is nonempty iff $\beta$ is coherent.

Example 3. Let $\Phi$ and $\beta_{1}: \Phi \rightarrow[0,1]$ be as in Example 2. Thus, $\beta_{1}(p)=1 / 2$ and $\beta_{1}(p \vee q)=2 / 3$. The vertices (extreme points) of the rectangle $\mathscr{R}_{\beta_{1}}$ are easy to compute: $v_{1}=(2 / 3,1 / 2) ; v_{2}=(0,1 / 2) ; v_{3}=(0,1) ; v_{4}=(2 / 3,1)$. Thus, $\mathscr{C}_{\beta_{1}}=\mathscr{C}_{\Phi} \cap \mathscr{R}_{\beta_{1}}$ coincides with the gray region as in Figure 3. 


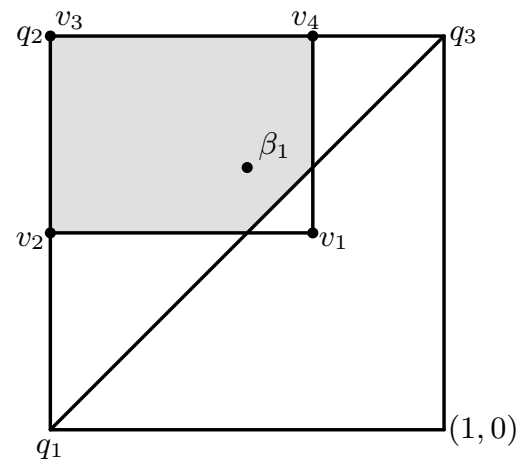

Fig. 3. The coherent book $\beta_{1}$ from Example 2 and the convex set $\mathscr{C}_{\beta_{1}}$ (the gray region) obtained by intersecting $\mathscr{C}_{\Phi}$ (the triangle of vertices $q_{1}, q_{2}$ and $q_{3}$ ) and the rectangle $\mathscr{R}_{\beta_{1}}$ (whose vertices are $v_{1}, v_{2}, v_{3}$ and $v_{4}$ ).

The following result shows that, for every fixed coherent book $\beta$, the convex set $\mathscr{C}_{\beta}$ characterizes all the coherent books which are jointly coherent with $\beta$.

Proposition 2. Let $\beta, \beta^{\prime}: \Phi \rightarrow[0,1]$ be two coherent books. Then the following conditions are equivalent:

1. $\beta^{\prime}$ is jointly coherent with $\beta$;

2. $\beta^{\prime} \in \mathscr{C}_{\beta}$.

Proof. (1) $\Rightarrow(2)$. Assume that $\beta$ and $\beta^{\prime}$ are jointly coherent. Since $\beta^{\prime}$ is coherent then, by Theorem $1, \beta^{\prime} \in \mathscr{C}_{\Phi}$. We only have to show that $\beta^{\prime} \in \mathscr{R}_{\beta}$. Suppose, by contradiction, that $\beta^{\prime} \notin \mathscr{R}_{\beta}$, i.e. there exists $1 \leq i \leq k$ such that $\left|\beta_{i}-\beta_{i}^{\prime}\right|>d_{i}^{+}$ (or $\left|\beta_{i}-\beta_{i}^{\prime}\right|<d_{i}^{-}$, but the reasoning is analogous). The definition of $\mathscr{R}_{\beta}$ immediatly implies that $\beta^{\prime}$ is not coherent, a contradiction.

$(2) \Rightarrow(1)$. Let $\beta^{\prime} \in \mathscr{C}_{\beta}=\mathscr{C}_{\Phi} \cap \mathscr{R}_{\beta}$, i.e. $\beta^{\prime}$ is a coherent book which satisfies the above conditions 1.-3. Let $\alpha$ be any book in $\Xi\left(\beta, \beta^{\prime}\right)$. Since $\beta$ is coherent, we have that $\beta \in \mathscr{C}_{\beta}$ and, by assumption, $\beta^{\prime} \in \mathscr{C}_{\beta}$, which together imply that $\alpha \in \mathscr{C}_{\beta}$. Thus $\alpha \in \mathscr{C}_{\Phi}$, which, by Theorem 1, implies that is coherent, therefore $\beta$ and $\beta^{\prime}$ are jointly coherent books.

It is immediate to see that the relation of being jointly coherent is symmetric. Therefore, from Proposition 2 above $\beta$ and $\beta^{\prime}$ are jointly coherent iff $\beta^{\prime} \in \mathscr{C}_{\beta}$ iff $\beta \in \mathscr{C}_{\beta^{\prime}}$. Therefore the following is immediate.

Corollary 1. Let $\beta, \beta^{\prime}: \Phi \rightarrow[0,1]$ be two coherent books. Then $\beta$ and $\beta^{\prime}$ are jointly coherent iff $\beta, \beta^{\prime} \in \mathscr{C}_{\beta} \cap \mathscr{C}_{\beta^{\prime}}$.

In the next we will show, for every coherent book $\beta$, another geometric characterization of $\mathscr{C}_{\beta}$. Recalling that every closed convex subsets of $\mathbb{R}^{k}$ is an intersection of halfspaces (see $[4$, Theorem 3.8$]$ ), for every polytope $\mathscr{P} \subseteq \mathbb{R}^{k}$, there 
are linear polynomials $f_{1}, \ldots, f_{n}$ such that $\mathscr{P}=\left\{\left(x_{1}, \ldots, x_{k}\right) \in \mathbb{R}^{k} \mid \forall i=\right.$ $\left.1, \ldots, n, f_{i}\left(x_{1}, \ldots, x_{k}\right) \geq 0\right\}$. In what follows, without danger of confusion, for every finite set of events $\Phi=\left\{e_{1}, \ldots, e_{k}\right\}$, we will write $f_{1}, \ldots, f_{n}$ for those polynomials such that

$$
\mathscr{C}_{\Phi}=\left\{\left(x_{1}, \ldots, x_{k}\right) \in \mathbb{R}^{k} \mid \forall i=1, \ldots, n, f_{i}\left(x_{1}, \ldots, x_{k}\right) \geq 0\right\} .
$$

For the sake of a lighter notation, we will denote by $K$ the index set $\{1, \ldots, k\}$.

Let us fix two points $a=\left(a_{1}, \ldots, a_{k}\right)$ and $b=\left(b_{1}, \ldots, b_{k}\right)$ of $\mathbb{R}^{k}$, and a subset $J$ of $K$. Then, we denote by $\left(a_{J}, b_{K \backslash J}\right)$ the tuple obtained by substituting $b_{j}$ by $a_{j}$, in $b$, for each $j \in J$.

Theorem 2. Let $\mathscr{C}_{\Phi}, f_{1}, \ldots, f_{n}$ be as in (4) and let $\beta, \beta^{\prime}$ be two coherent books. Then, $\beta^{\prime} \in \mathscr{C}_{\beta}$ iff $\beta^{\prime}$ is solution of the following system,

$$
\mathscr{S}(\beta)=\left\{f_{i}\left(\beta_{J}, x_{K \backslash J}\right) \geq 0 \mid i=1, \ldots, n, J \subseteq K\right\} .
$$

In other words, $\mathscr{C}_{\beta}$ coincides with the set of solutions of $\mathscr{S}(\beta)$.

Proof. $(\Leftarrow)$. Assume, by contraposition, that $\beta^{\prime} \notin \mathscr{C}_{\beta}$. Thus, by Proposition $2, \beta$ and $\beta^{\prime}$ are not jointly coherent and hence, by Remark 1 , there exists a $\hat{\beta} \in \Xi\left(\beta, \beta^{\prime}\right)$ which is not coherent. This means, by Theorem 1 , that $\hat{\beta} \notin \mathscr{C}_{\Phi}$. Therefore, by (4), there exists an index $i \in\{1, \ldots, n\}$ such that $f_{i}\left(\hat{\beta}_{1}, \ldots, \hat{\beta}_{k}\right)<$ 0 and in particular $\hat{\beta}$ is not a solution of $\mathscr{S}(\beta)$.

$(\Rightarrow)$. Assume, again by contraposition, that $\beta^{\prime}$ is not solution of $\mathscr{S}(\beta)$. Thus, there exists a $J \subseteq K$ and an index $i \in\{1, \ldots, n\}$ such that $f_{i}\left(\beta_{J}, \beta_{K \backslash J}^{\prime}\right)<0$. Therefore, the claim immediately follows by observing that $\left(\beta_{J}, \beta_{K \backslash J}^{\prime}\right) \in \Xi\left(\beta, \beta^{\prime}\right)$ and $\left(\beta_{J}, \beta_{K \backslash J}^{\prime}\right) \notin \mathscr{C}_{\Phi}$ proving that $\beta$ and $\beta^{\prime}$ are not jointly coherent.

An immediate consequence of the above theorem is the decidability of the problem determining if two rational-books are jointly coherent. For the following result to make sense, we will hence assume that the books involved takes value into the rational unit interval $[0,1] \cap \mathbb{Q}$.

Corollary 2. Given two rational-valued books $\beta_{1}, \beta_{2} \in \mathscr{C}_{\Phi}$, the problem of determining if $\beta_{1}$ and $\beta_{2}$ are jointly coherent is decidable.

Proof. The following (sketched) procedure, which takes in input the events $e_{1}, \ldots, e_{k}$ and the rational numbers $\beta_{1}\left(e_{i}\right)$ 's and $\beta_{2}\left(e_{i}\right)$ 's, decides if $\beta_{1}$ and $\beta_{2}$ are jointly coherent.

Step 1: Determine the extremal points of $\mathscr{C}_{\Phi}$ by computing, for each truthassignment $h_{j}, q_{j}=\left(h_{j}\left(e_{1}\right), \ldots, h_{j}\left(e_{k}\right)\right)$ as in (1).

Step 2: Let $q_{t_{1}}, \ldots, q_{t_{r}}$ be, among the $q_{j}$ 's, the extremal points of a face $\mathscr{F}_{t}$ of $\mathscr{C}_{\Phi}$ and let $f_{t}\left(x_{1}, \ldots, x_{k}\right)$ the be equation of the hyperplane through $q_{t_{1}}, \ldots, q_{t_{r}}$.

Step 3: Iterate Step 2 for all faces $\mathscr{F}_{1}, \ldots, \mathscr{F}_{n}$ of $\mathscr{C}_{\Phi}$ and hence determine $f_{1}, \ldots, f_{n}$ such that $\mathscr{C}_{\Phi}=\left\{\left(x_{1}, \ldots, x_{k}\right) \in \mathbb{R}^{k} \mid \forall i=1, \ldots, n, f_{i}\left(x_{1}, \ldots, x_{k}\right) \geq\right.$ $0\}$ as in (4). 
Step 4: Introduce the system of inequalities $\mathscr{S}\left(\beta_{1}\right)$ as in the statement of Theorem 2 .

Therefore, in the end, check if $\beta_{2}=\left(\beta_{2}\left(e_{1}\right), \ldots, \beta_{2}\left(e_{k}\right)\right)$ is a (rational) solution of $\mathscr{S}\left(\beta_{1}\right)$.

Our last example applies the result of Theorem 2 and exemplifies also the procedure sketched in the proof of Corollary 2.

Example 4. Let $\Phi=\{p, p \vee q\}$ and $\beta_{1}(p)=\frac{1}{2}, \beta_{1}(p \vee q)=\frac{2}{3}$. Then

$$
\mathscr{C}_{\Phi}=\left\{\left(x_{1}, x_{2}\right) \in \mathbb{R}^{2}: 0 \leq x_{1}, x_{2} \leq 1, x_{1} \leq y_{2}\right\} .
$$

A book $\beta$ is jointly coherent with $\beta_{1}$ if and only if it satisfies the system given by the following inequalities

- for $J=\emptyset$, we have $x_{1} \geq 0,1-x_{1} \geq 0, x_{2} \geq 0,1-x_{2} \geq 0$ and $x_{2}-x_{1} \geq 0$;

- for $J=\{1\}$, we have $1 / 2 \geq 0,1-1 / 2 \geq 0, x_{2} \geq 0,1-x_{2} \geq 0$ and $x_{2}-1 / 2 \geq 0$

- for $J=\{2\}$, we have $x_{1} \geq 0,1-x_{1} \geq 0,2 / 3 \geq 0,1-2 / 3 \geq 0$ and $2 / 3-x_{1} \geq 0$

- for $J=\{1,2\}$, we have $1 / 2 \geq 0,1-1 / 2 \geq 0,2 / 3 \geq 0,1-2 / 3 \geq 0$ and $2 / 3-1 / 2 \geq 0$.

Notice that the inequalities obtained for $J=\emptyset$ just describes $\mathscr{C}_{\Phi}$ and it is also immediate to see that for $J=\{1,2\}$, we get the inequalities which assure that $\beta_{1}$ is a coherent book.

\section{Conclusion and future work}

The present work is motivated by the observation that when two different bookmakers assign betting quotes over the same set of events, the notion of coherence is not enough to prevent a gambler who is allowed to bet on both assignments from a sure-win opportunity. We thereby proposed the notion of joint coherence of two books.

Our main results consist of geometrical characterizations of the space of books which are jointly coherent with a given one. Such a space is a closed convex subset of the set of all coherent books and it identifies which books can be considered "safe" once $\beta$ has been fixed.

We believe that the mathematics of joint coherence as well as its computational aspects deserve further attention. In particular, since (two) jointly coherent books are necessarily coherent, we wonder what is the effect of the property of being jointly coherent on the sets of probability measures which extend them, by de Finetti's theorem, on the Boolean algebra generated by the events. Further, still on this line, it would be interesting to extend the notion of joint coherence to more general theories of uncertainty and, in particular, to Walley's definition of coherence for imprecise probabilities where negative betting rates are forbidden [13]. 
We are interested also in providing different characterizations of the notion of joint coherence. In particular, following [7], where it is shown that (strict) coherence admits three characterizations (algebraic, geometrical and logical), one of the aims for the future is to extend such characterizations to joint coherence as well.

A very natural question that computer scientists may rise is whether it is possible to establish a computational bound to the problem of determining whether two books are jointly coherent. Although we proved that checking joint coherence of two books is decidable, providing a NP-bound for the same seems challenging and it will be object of further investigation.

Joint coherence arises from allowing a multiplicity of bookmakers publishing coherent books, who can be viewed as individually rational agents. Grounding on this consideration, it is reasonable to think that this notion may suggest an alternative way to approach collective judgments (see for instance [3]) and collective rationality. This will also be addressed in our future work.

\section{Acknowledgments}

The first author acknowledges the support of the European Research Council, ERC Starting Grant GA:639276: "Philosophy of Pharmacology: Safety, Statistical Standards, and Evidence Amalgamation". Flaminio acknowledges partial support by the Spanish Ramon y Cajal research program RYC-2016-19799; the Spanish FEDER/MINECO project TIN2015- 71799-C2-1-P and the SYSMICS project (EU H2020-MSCA-RISE-2015, Project 689176).

\section{References}

1. B. de Finetti. Sul significato soggettivo della probabilità. Fundamenta Mathematicae, 17:289-329, 1931.

2. B. de Finetti. Theory of Probability, vol. 1. John Wiley and Sons, New York, 1974.

3. F. Dietrich, C. List. Probabilistic opinion pooling. The Oxford Handbook of Probability and Philosophy (A. Hájek and C. Hitchcock editors), 2016.

4. G. Ewald, Combinatorial Convexity and Algebraic Geometry. Springer-Verlag New York, 1996.

5. T. Flaminio, L. Godo, H. Hosni. On the logical structure of de Finetti's notion of event. Journal of Applied Logic 12(3): 279-301, 2014.

6. T. Flaminio, H. Hosni, F. Montagna. Strict Coherence on Many-Valued Events. Journal of Symbolic Logic 83(1): 55-69, 2018.

7. T. Flaminio. Three characterizations of strict coherence on infinite-valued events. Submitted.

8. D. Mundici, Bookmaking over infinite-valued events. International Journal of Approximate Reasoning 43(3): 223-240, 2006.

9. D. Mundici, De Finetti coherence and the product law for independent events. Synthese 196(1): 265-271, 2019.

10. R. Nau, K. F. MaCardle. Coherent Behavior in Noncooperative Games. Journal of Economic Theory 50: 424-444, 1990. 
11. R. Nau. Joint Coherence in Games of Incomplete Information. Management Sciences 38(3): 374-387, 1992.

12. J. Paris. A note on the Dutch Book method. Proceedings of the Second International Symposium on Imprecise Probabilities and their Applications (G. De Cooman, T. Fine, and T. Seidenfeld, editors), ISIPTA 2001, Shaker Publishing Company, Ithaca, NY, USA: 301-306, 2001.

13. P. Walley. Statistical Reasoning with Imprecise Probabilities, London: Chapman and Hall, 1991. 